\title{
Power law and small world properties in a comparison of traffic city networks
}

\author{
MA Ke ${ }^{1,2^{*}}$, WANG ZhongWen ${ }^{3}$, JIANG Jian ${ }^{4}$, ZHU GuangXi ${ }^{2} \&$ LI Wei $^{5}$ \\ ${ }^{1}$ Department of Physics, College of Maths and Physics, China University of Geosciences, Wuhan 430074, China; \\ ${ }^{2}$ Department of Electronics and Information Engineering, Huazhong University of Science and Technology, Wuhan 430074, China; \\ ${ }^{3}$ Department of Mathematics, Kashgar Teachers College, Kashgar 844006, China; \\ ${ }^{4}$ Institut Superieur des Materiaux du Mans, 44, Avenue F.A. Bartholdi, 72000 Le Mans, France; \\ ${ }^{5}$ Institute of Particle Physics, Central China Normal University, Wuhan 430079, China
}

Received May 30, 2011; accepted July 6, 2011

\begin{abstract}
We analyze the statistical properties of the urban public bus networks of two cities (Beijing and Chengdu) in China. To this end, we present a comprehensive survey of the degree distribution, average path length, and clustering of both networks. It is shown that both networks exhibit small world behavior and are hierarchically organized. We also discuss the differences between the statistical properties displayed by the two networks. In addition, we propose a weight distribution approach to study the passenger flow through the public bus networks we considered. A hierarchical structure is observed here also.
\end{abstract}

public transport network, complex network, power-law distribution, small-world

Citation: $\quad$ Ma K, Wang Z W, Jiang J, et al. Power law and small world properties in a comparison of traffic city networks. Chinese Sci Bull, 2011, 56: 3731-3735, doi: $10.1007 / \mathrm{s} 11434-011-4769-4$

Solving the problem of traffic congestion is of great importance for the safety and convenience of modern society [1-6]. Recently, empirical evidence has shown that many transportation systems can be described by complex networks characterized by the small world [7] and/or scale-free properties [8]. The small-world effect became so known as it provides an elegant explanation for Milgram's experiment of the six degrees of separation [9]. The first suitable model capable of explaining the small-world effect was reported by Watts and Strogatz [7] which has motivated several applications to real-world systems.

In recent years, several public transport systems (PTS) have been investigated using various concepts of the statistical physics of complex networks [10-20]. Among them, many studies have focused on the complexity of urban public transport systems. Latora and Marchiori [19,21] introduced the definition of efficiency coefficient and applied it to a study of the Boston subway. Sen et al. [22] concluded

*Corresponding author (email: mark@iopp.ccnu.edu.cn) that India's railway network exhibited small world properties. Similar properties were reported by Seaten and Hackett [23] in a study of railway networks in Boston and Vienna. Jiang and Claramunt [24] found that the topological networks of streets exhibited small world properties but were not scale-free. Guimera et al. [25] showed that the worldwide air traffic network was a scale-free and small world network. Wang et al. [26] proposed a hierarchical geographical model to mimic a real traffic system, upon which a random walk generates a power-law-like travel displacement distribution with tunable exponent. $\mathrm{Li}$ and Cai [16] showed that the topological structure of the air traffic network of China (ANC) had two key characteristics of smallworld networks, a short average path-length and a high degree of clustering. He et al. [27,28] investigated the urban transport networks of four cities in China and introduced a model whose numerical results can fit the empirical data well. Wu et al. [29] concluded that the urban transit system in Beijing is a scale-free network. Zhao et al. [30] introduced three kinds of models to study the properties of the 
public transport in Beijing. Zhu et al. [31] investigated the scaling of directed dynamical small-world networks with random responses. Some other noteworthy results can be seen in [32-40].

In this paper, we construct the public bus network based on the data for two Chinese cities, Chengdu $(N=220, E$ $=12280$, where $N$ is the number of bus stops and $E$ is the number of bus lines between two stops, www.chengdu. 8684.cn) and Beijing ( $N=498, E=29450$, www.bjbus.com). Three most robust measures of network topology: degree distribution, characteristic path length and clustering coefficient have been checked, as well as the passenger flow analysis. Despite a large difference in size of the considered networks they share several universal features.

\section{Comparison of fundamental characteristics of networks}

The degree distribution is one of the three most robust measures of network topology. The degree of a node is the number of edges connected with this node. The degree $k_{i}$ of node $i$ can be defined as $k_{i}=\sum_{i \in G} a_{i, j} \quad\left(a_{i, j}\right.$ is the adjacency of connection among nodes). The average degree is $\bar{k}=\sum_{i \in G} k_{i} / N=2 K / N \quad(K$ is the total number of links in the network, and $N$ is the total number of nodes). The way the degree is distributed among the nodes is an important property of a network that can be investigated by calculating the degree distribution $P(k)$, i.e. the probability of finding nodes with $k$ links. The degree distribution is defined as $P(k)=N(k) / N$, where $N(k)$ is the number of nodes with $k$ links. The majority of papers about complex networks have shown that in most of the real systems the degree distribution follows a power law for large $k: P(k) \sim N(k) \sim k^{-\gamma}$ with the exponent between 2 and 3 [41-43]. Networks with such a degree distribution are called scale-free [43]. The results found are in contrast with what are expected for random graphs [44]. In fact, a random graph with $N$ nodes and $K$ edges (an average of $\bar{k}$ per node), i.e. a graph obtained randomly selecting the $K$ couples of nodes to be connected, exhibits a Poisson degree distribution centered at $\bar{k}$.

The comparison of degree distribution of the public bus networks of Beijing and Chengdu is presented in Figure 1. The power-law-tail behavior indicates that both networks have scale-free topology. The consequence of heavy tails is that the average behavior of the system is not typical [45]. There is overwhelming evidence that human activities including traffic networks are characterized by heavy-tailed statistics [46-49]. The exponent of degree distribution for Beijing is 3.7, much smaller than that for Chengdu. This result shows there are comparatively more nodes with high degrees in Beijing than in Chengdu, where it is 6.1. The

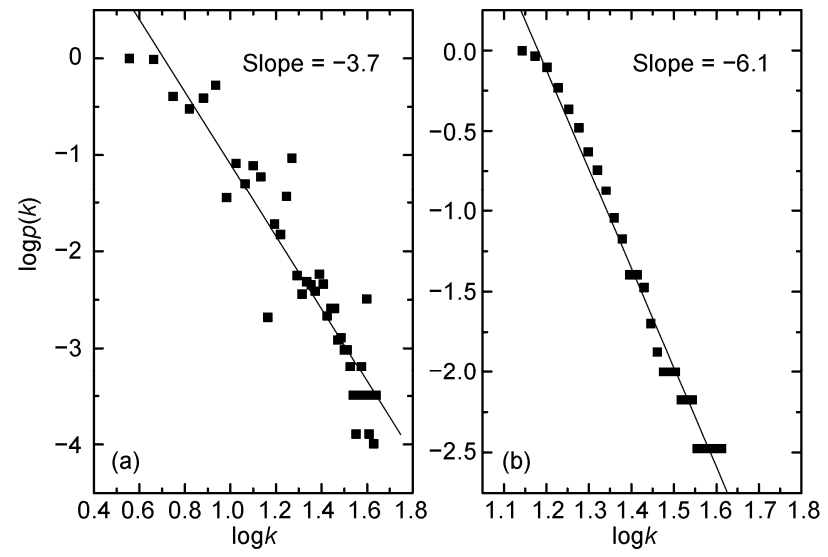

Figure 1 Comparison of the degree distributions of the public bus networks of cities Beijing (a) and Chengdu (b) in China. $P(k)$ is the degree distribution function, and $k$ is the degree of each node. A power-law distribution is obviously shown in the log-log plot and the fitting curve with black solid line matches the data well.

reason is that more hub bus stations exist in the Beijing public bus network.

Another robust measure of network topology is characteristic path length. Analysis of many networks has shown similar properties: in most real-world networks it is possible to reach any node from another one, going through a number of edges that is small compared with the total number of existing nodes in the system [9]. The typical separation between two generic nodes in a graph $G$ can be measured by the characteristic path length $L$, defined as

$$
L=\frac{1}{N(N-1)} \sum_{i, j \in G, i \neq j} d_{i, j} \text {, }
$$

where $d_{i, j}$ is the length of the shortest path between nodes $i$ and $j$, i.e. the minimum number of edges covered to go from $i$ to $j$. Clustering is the third robust measure of network topology. In many real-world networks, for instance in social systems there is a high probability that two individuals linked by an acquaintance have a third acquaintance in common. Such tendency can be measured by the clustering coefficient $C$. For each node $i$ of $G$, we consider the subgraph $G_{i}$ of first neighbors that is obtained in two steps: (1) extracting $i$ and its first neighbors from $G$; (2) removing the node $i$ and all the incident edges. If node $i$ has $k_{i}$ neighbors, then $G_{i}$ will have $k_{i}$ nodes and at most $k_{i}\left(k_{i}-1\right) / 2$ edges. $C_{i}$ is proportional to the fraction of these edges that really exist and measures the local group cohesiveness of vertex $i . C$ is the average of $C_{i}$ calculated over all nodes:

$$
C(G)=\left\langle C_{i}\right\rangle=\frac{1}{N} \sum_{i \in G} C_{i},
$$

where

$$
C(i)=\frac{2 e_{i}}{k_{i}\left(k_{i}-1\right)} \sum_{j, m} \frac{a_{i, j} a_{j, m} a_{m, j}}{k_{i}\left(k_{i}-1\right)},
$$

where $e_{i}$ is the number of edges in $G_{i}$. By definition, $C$ takes values in the interval $[0,1]$. Notice that $C$ is related to the 
number of triangles present on the network. A detailed description of the network can be obtained by the distribution of $C_{i}$ among the nodes of the network. For instance, important information can be extracted by considering $C(k)$, the average clustering coefficient restricted to classes of vertices of degree $k$. In many cases $C(k)$ exhibits a power law decay as a function of $k$, i.e. a hierarchy with low degree vertices belonging to well interconnected communities and hubs connecting many vertices not directly connected to each other.

Random graphs have a characteristic path length $L$ that grows only logarithmically with $N$, and a clustering coefficient $C=\langle k\rangle=N$ going to zero for large $N$. Conversely, a regular lattice has a finite clustering coefficient and a characteristic path length $L$ which grows linearly with $N$. Watts and Strogatz have shown that many real-world networks have properties intermediate between random graphs and regular lattices. All such networks that have been named small worlds have at the same time: (1) a small characteristic path length as in random graphs; and (2) a large clustering coefficient, typical of regular lattices. To check whether a network is a small world it has been proposed to compare the value of $L$ and $C$ with those obtained for the randomized version of the network, i.e. for a network with the same $N$ and $K$ and in which the edges are randomly distributed with a uniform probability, among all the nodes. In a small-world network $L \sim L_{\text {rand }}$ and $C>>C_{\text {rand }}$.

Therefore, we calculate the average path length and the clustering coefficient of the public bus networks of Beijing and Chengdu. The results in Table 1 are compared with those obtained from random graphs with the same number of nodes and links. Though both networks have a small average path length, Chengdu's public bus network has the much smaller average path length in that any two bus stops can be connected in just two steps. In addition, the two networks have $C \gg>C_{\text {rand }}$. The smaller $C$ of Chengdu's public bus network compared with Beijing's public bus network is because there are fewer bus hub stations in Chengdu than there are in Beijing.

The correlation between the cluster coefficient $C(k)$ and the degree $k$ of two public bus networks is also investigated (Figure 2). A scale-free behavior is clearly emerging in Figure 2: (1) the power law fit yields exponents -0.65 and -0.75 for Beijing and Chengdu, respectively; (2) both exponents are significantly larger for large $N$ than the random network prediction $C(k) \sim k^{-1}[44,50]$. Moreover, the negative correlations account for the hierarchy of the public bus

Table 1 The coefficient of the public bus networks of Beijing and Chengdu in China

\begin{tabular}{lccccc}
\hline & $C$ & $L$ & $C_{\text {rand }}$ & $L_{\text {rand }}$ & $K$ \\
\hline Chengdu & 0.14 & 1.9 & 0.005 & 1.7 & 111.6 \\
Beijing & 0.21 & 6.6 & 0.002 & 5.1 & 118.3 \\
\hline
\end{tabular}
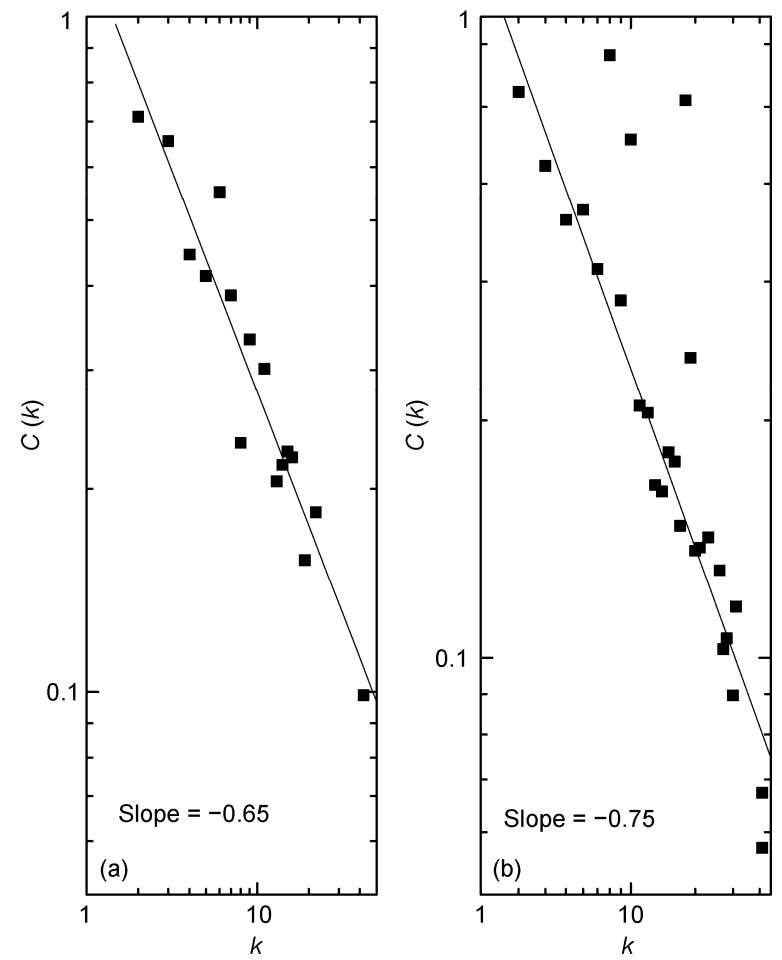

Figure 2 Comparison of the correlation between the cluster coefficient and degree of two public bus networks of cities Beijing (a) and Chengdu (b) in China. $C(k)$ is the cluster coefficient, and $k$ is the degree. From the straight lines, we find that there is negative correlation between the two quantities in each panel. As the slopes are -0.65 and -0.75 respectively, the degrees of correlation in the two panels are almost equal.

networks we considered. Many real-life networks including the Internet, World Wide Web, and the actor network, are characterized by the existence of a hierarchical structure [51-53], which can usually be detected by the negative correlation between the clustering coefficient and the degree. All the general small-world-properties mentioned above are simultaneously present in our public bus networks.

As an obvious human activity, the passenger flow is the main purpose of the PTS and naturally reflects its significant features. We investigate the passenger flow on our public bus networks. The weight $w_{i, j}$ of a link between stations $i$ and $j$ is taken to be the sum of passenger flows in both directions on the link, i.e. $i \rightarrow j$ and $j \rightarrow i$. The strength $s_{i}$ of station $i$ is then defined to be the sum $s_{i}=\sum_{j=1}^{N} w_{i, j}$.

Figure 3 shows the distributions of passenger flow in the two public bus networks. It is observed that the weight distribution $P(S)$ exhibits power-law behavior with exponents of -2.2 and -2.1 , respectively. Despite a significant difference in passenger flow size, such nearly similar passenger flow distribution exponents account for the uniform hierarchical structure, as previously found and discussed, of passenger flows in both Beijing and Chengdu's public bus network. That is not unexpected, because as the metropolises of China, Beijing and Chengdu's bus stops are located in 

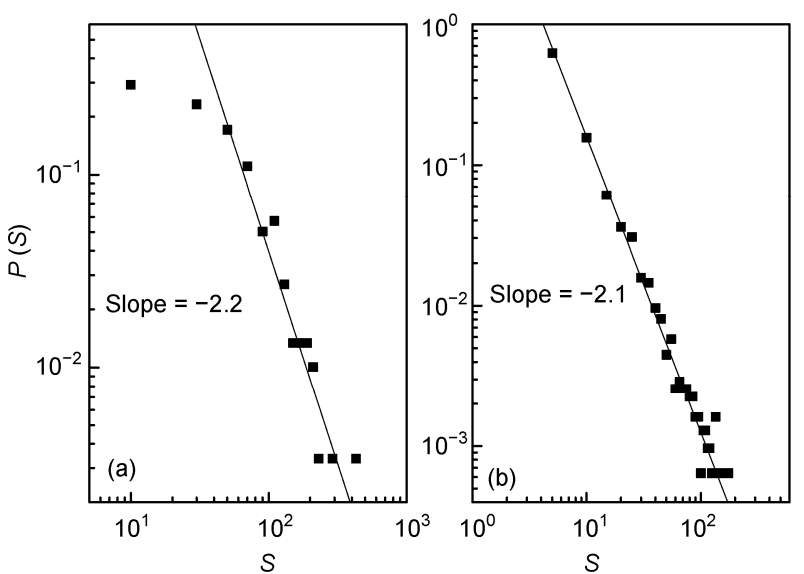

Figure 3 Comparison of the distribution of weights for the public bus networks of cities Beijing (a) and Chengdu (b) in China. $P(S)$ is the weight function, and $S$ is the weight. (a) There is a power law distribution in most of the observed range of weight and the slope of fitting straight line is -2.2 ; (b) a power law distribution is also observed, and the slope is -2.1 .

a similar way. For example, most facilities are located near stations, so each station naturally has an abundance of passengers.

\section{Conclusions}

We have constructed the public bus network based on the data of two Chinese cities (Beijing and Chengdu). The public bus networks under consideration appear to be strongly correlated small-world and hierarchical structures with powerlaw distribution of degree, high values of clustering coefficients (especially in $L$ and less in $C$-spaces) and comparatively low average shortest path values. It is found that the clustering coefficient has negative correlation with the numbers of the bus lines that pass that stop. In particular we have considered the passenger flow on two cities' public bus networks by the weight denoting the number of passengers through a link between stations. The weights characterizing the passenger flow also exhibit a hierarchical structure feature with power-law distribution behavior. The comparison of statistical properties between Beijing and Chengdu in detail shows more bus hub stations in Beijing than that in Chengdu, therefore a higher efficiency public bus network of Beijing. These results may contribute to the description of the hierarchies and organizational principles for the public transport networks.

This work was supported by the National Natural Science Foundation of China (10747003).

1 Nagel K, Schreckenberg M. A cellular automaton model for freeway traffic. J Phys I France, 1992, 2: 2221-2229

2 Helbing D, Huberman B A. Coherent moving states in highway traffic. Nature, 1998, 396: 738-740

3 Kerner B S. The Physics of Traffic. Berlin: Springer, 2004
4 Biham O, Middleton A, Levine D. Self-organization and a dynamical transition in traffic-flow models. Phys Rev A, 1992, 46: 6124-6127

5 Chung K H, Hui P M, Gu G Q. Two-dimensional traffic flow problems with faulty traffic lights. Phys Rev E, 1995, 51: 772-774

6 Angel O, Holroyd A E, Martin J B. The jammed phase of the Biham-Middleton-Levine traffic model. Elec Comm In Prob, 2005, 10: $167-178$

7 Watts D J, Strogatz S H. Collective dynamics of small-world networks. Nature, 1998, 393: 440-442

8 Albert R, Jeong H, Barabasi A L. Internet: Diameter of the worldwide web. Nature, 1999, 401: 130-131

9 Milgram S. The small world problem. Psychology Today, 1967, 2: 60-67

10 Matheus P V, Luciano F C. How many nodes are effectively accessed in complex networks? Phys Lett A, 2001, 375: 2085-2088

11 Barthelemy M. Spatial networks. Phys Rep, 2011, 499: 1-101

12 Scellato S, Fortuna L, Frasca M, et al. Traffic optimization in transport networks based on local routing. Eur Phys J B, 2010, 73 : 303-308

13 Li G, Reis S D, Moreira A A, et al. Towards design principles for optimal transport networks. Phys Rev Lett, 2010, 104: 018701

14 Bagler G. Analysis of the airport network of India as a complex weighted network. Physica A, 2008, 387: 2972-2980

15 Albert R, Albert I, Nakarado G L. Structural vulnerability of the North American power grid. Phys Rev Letts, 2004, 69: 025103

16 Li W, Cai X. Statistical analysis of airport network of china. Phys Rev E, 2004, 69: 046106

17 Barrat A, Barthelemy M, Pastor-Satorras R, et al. The architecture of complex weighted networks. Proc Natl Acad Sci USA, 2004, 101: 3747-3752

18 Strogatz S H. Exploring complex networks. Nature, 2001, 410: 268-276

19 Latora V, Marchiori M. Efficient behavior of small-world networks. Phys Rev Lett, 2001, 87: 1-4

20 Amaral L A, Scala A, Barthelemy M, et al. Classes of small-world networks. Proc Natl Acad Sci USA, 2000, 97: 11149-11152

21 Latora V, Marchiori M. Is the Boston subway a small-world network? Physica A, 2002, 314: 109-113

22 Sen P, Dasgupta S, Chatterjee A. Small-world properties of the Indian railway network. Phys Rev E, 2003, 67: 036106

23 Seaton K A, Hackett L. Stations, trains and small-world networks. Physica A, 2004, 339: 635-644

24 Jiang B, Claramunt C. Topological analysis of urban street networks. Environ Plan B, 2004, 31: 151-162

25 Guimera R, Mossa S, Turtschi A, et al. The worldwide air transportation network: Anomalous centrality, community structure, and cities' global roles. Proc Natl Acad Sci USA, 2005, 102: 7794-7799

26 Han X P, Hao Q, Wang B H, et al. Origin of the scaling law in human mobility: Hierarchy of traffic systems. Phys Rev E, 2011, 83: 036117

27 Chen Y Z, Fu C, Chang H, et al. Connectivity correlations in three topological spaces of urban bus-transport networks in China. Chin Phys B, 2008, 17: 3580-3587

28 Chen Y Z, Li N, He D. A study on some urban bus transport networks. Physica A, 2004, 376: 747-751

29 Wu J J, Gao Z Y, Sun H J. Urban transit system as a scale-free network. Mod Phys Letts B, 2004, 18: 1043-1049

30 Zhao J S, Di Z R, Wang D H. Complexity and efficiency of Beijing transit network. Comp Syst Comp Sci, 2005, 2: 45-48

31 Zhu C, Xiong S, Tian Y, et al. Scaling of directed dynamical small-world networks with random responses. Phys Rev Letts, 2004, 92: 218702

32 Dai J C, Li X. Multi-agent systems for simulating traffic behaviors. Chinese Sci Bull, 2010, 55: 293-300

33 Sun X Y, Wang B H, Yang H X, et al. Effects of information feedback on an asymmetrical two-route scenario. Chinese Sci Bull, 2009, 54: 3211-3214

34 Zang C X, Huang B C, Li Z Y, et al. Magnetic properties of highroad-side pine tree leaves in Beijing and their environmental significance. Chinese Sci Bull, 2006, 51: 3041-3052 
35 Tang $\mathrm{T}$ Q, Huang $\mathrm{H}$ J. Continuum models for freeways with two lanes and numerical tests. Chinese Sci Bull, 2004, 49: 2097-2104

36 Jiang R, Wu Q S, Zhu Z J. A new dynamics model for traffic flow. Chinese Sci Bull, 2001, 46: 345-349

37 Li Q, Chen X, Chen J, et al. An evacuation risk assessment model for emergency traffic with consideration of urban hazard installations. Chinese Sci Bull, 2010, 55: 1000-1006

38 Zhang Y, Fang B X, Zhang Y Z. Identifying heavy hitters in high-speed network monitoring. Sci China Inf Sci, 2010, 53: 659-676

39 Sun X L, Jia L M, Dong H H, et al. Urban expressway traffic state forecasting based on multimode maximum entropy model. Sci China Tech Sci, 2010, 53: 2808-2816

$40 \mathrm{Xu} \mathrm{M}$, Gao Z Y. A complementary formulation for traffic equilibrium problem with a new nonadditive route cost. Sci China Tech Sci, 2010, 53: 3264-3274

41 Satorras P, Vespignani R. Modeling the world-wide airport network. Eur Phys J B, 2008, 38: 205-210

42 Fredrik L, Christofer R E, Luis A, et al. The web of human sexual contacts. Nature, 2001, 411: 907-908

43 Albert R, Barabasi M. Statistical mechanics of complex networks.
Rev Mod Phys, 2002, 74: 47-97

44 Erdos P, Renyi A. On random graphs. Publ Math Debrecen, 1959, 6: 290-297

45 Montis A D, Barthelemy M, Chessa A, et al. The structure of interurban traffic: A weighted network analysis. Environ Plan, 2007, 34: 905-924

46 Barabasi A L. The origin of bursts and heavy tails in human. Nature, 2005, 435: 207-211

47 Sanwalani V, Clauset A, Moore C. Scale invariance in road networks. Phys Rev E, 2006, 73: 026130

48 Jung W S, Wang F Z, Stanley H E. Gravity model in the Korean highway. Europhys Lett, 2008, 81: 48005

49 Zhou T, Wang B H. Role of activity in human dynamics. Europhys Lett, 2008, 82: 28002

50 Bollobas B. Random Graphs. London: Academic Press, 1985

51 Ravasz E, Barabasi A L. Hierarchical organization in complex networks. Phys Rev E, 2003, 67: 026112

52 Trusina A, Maslov S, Minnhagen P, et al. Hierarchy measures in complex networks. Phys Rev Lett, 2004, 92: 178702

53 Kim B J. Geographical coarse graining of complex networks. Phys Rev Lett, 2004, 93: 168701

Open Access This article is distributed under the terms of the Creative Commons Attribution License which permits any use, distribution, and reproduction in any medium, provided the original author(s) and source are credited. 